\title{
Correlations between genetic, epigenetic and phenotypic variation of an introduced clonal herb
}

\author{
Mo-Zhu Wang ${ }^{1,2,3} \cdot$ Hong-Li Li $i^{3}$ Jun-Min $\mathrm{Li}^{2} \cdot$ Fei-Hai Yu $\mathbb{\circledR}^{1,2}$
}

Received: 13 May 2019 / Revised: 6 July 2019 / Accepted: 30 July 2019 / Published online: 20 August 2019

(c) The Author(s), under exclusive licence to The Genetics Society 2019

\begin{abstract}
Heritable epigenetic modifications may occur in response to environmental variation, further altering phenotypes through gene regulation, without genome sequence changes. However, epigenetic variation in wild plant populations and their correlations with genetic and phenotypic variation remain largely unknown, especially for clonal plants. We investigated genetic, epigenetic and phenotypic variation of ten populations of an introduced clonal herb Hydrocotyle vulgaris in China. Populations of $H$. vulgaris exhibited extremely low genetic diversity with one genotype exclusively dominant, but significantly higher epigenetic diversity. Both intra- and inter-population epigenetic variation were related to genetic variation. But there was no correlation between intra-/inter-population genetic variation and phenotypic variation. When genetic variation was controlled, intra-population epigenetic diversity was related to petiole length, specific leaf area, and leaf area variation, while inter-population epigenetic distance was correlated with leaf area differentiation. Our study provides empirical evidence that even though epigenetic variation is partly under genetic control, it could also independently play a role in shaping plant phenotypes, possibly serving as a pathway to accelerate evolution of clonal plant populations.
\end{abstract}

\section{Introduction}

In natural ecosystems, plants constantly experiencing environmental changes often develop various mechanisms to cope with habitat heterogeneity (Grativol et al. 2012; Bian et al. 2013; Schulz et al. 2014). In addition to the adaptation of generating new traits by genetic changes, phenotypic plasticity provides an alternative regulation (Boyko and Kovalchuk 2011; Schulz et al. 2014; Banta and Richards 2018). More recently, epigenetic modification has

Supplementary information The online version of this article (https:// doi.org/10.1038/s41437-019-0261-8) contains supplementary material, which is available to authorised users.

$\triangle$ Fei-Hai Yu

feihaiyu@126.com

1 Institute of Wetland Ecology \& Clone Ecology, Taizhou University, Taizhou 318000, China

2 Zhejiang Provincial Key Laboratory of Plant Evolutionary Ecology and Conservation, Taizhou University, Taizhou 318000, China

3 School of Nature Conservation, Beijing Forestry University, Beijing 100083, China been widely considered as another candidate mechanism between random genetic and environmental variation accounting for plant phenotypes (Richards et al. 2017). Epigenetic mechanisms such as DNA methylation, histone modifications and small RNAs can cause stable alterations in gene expression without changing underlying DNA sequences (Verhoeven et al. 2010; Massicotte et al. 2011; Grativol et al. 2012). Nowadays, the best-studied epigenetic modification is DNA methylation, which is mostly the addition of a methyl group deriving from S-adenosyl-Lmethionine to the $\mathrm{C} 5$ position of a cytosine residue, catalysed by DNA methyltransferase (Cervera et al. 2002; Bossdorf et al. 2008). For plants, DNA methylation mainly occurs in three different sequence contexts, symmetric CG and $\mathrm{CHG}$ sites $(\mathrm{H}=\mathrm{A}, \mathrm{C}, \mathrm{T})$ and asymmetric $\mathrm{CHH}$ sites in transposable elements and repetitive sequences related to transcriptional repression (Schulz et al. 2014).

Variation in DNA methylation may arise from genetic control, environmental induction or spontaneous epimutations, affecting gene expression to regulate plant phenotypes (Herrera and Bazaga 2011; Richards et al. 2017; Banta and Richards 2018). Such epigenetically induced phenotypic variation could be transiently reversible or transgenerationally heritable due to different stability of DNA methylation changes, which varies from liable within 
a generation to stably persistent in multiple generations through meiosis and/or mitosis (Herman et al. 2014). Different from genetic variation, epigenetic variation could be triggered by environmental cues directly, flexible to rapidly buffer environmental fluctuations or heritable to be further shaped by natural selection (Gao et al. 2010; Herman and Sultan 2016; Richards et al. 2017; Thiebaut et al. 2019). Thus, epigenetic variation may contribute to plant adaptation, and provides an alternative and accelerated pathway for ecological and evolutionary processes of plants (Bossdorf et al. 2008).

Recently, the role of epigenetic mechanisms in microevolution has attracted much attention, leading to the increasing number of studies on epigenetic variation and on epigenetic effects in natural environments, with investigating DNA methylation patterns of wild populations as the first step towards revealing the ecological importance of epigenetic variation (Rapp and Wendel 2005; Bossdorf et al. 2008; Richards et al. 2010; Herrera and Bazaga 2011; Hirsch et al. 2013; Busconi et al. 2015). However, different habitat conditions typically result in extensive genetic adaptation, complicating estimation of epigenetic effects on phenotypic responses of plants coping with environmental variation (Verhoeven et al. 2010). Therefore, another major concern is the dependence of epigenetic processes on genetic variation (Massicotte et al. 2011). Some studies argue that epigenetic variation is absolutely the direct downstream consequence of genetic changes, while others suggest that it is partly or completely autonomous from genetic control to provide an additional inheritance system for evolution (Riddle and Richards 2002; Richards 2006; Bossdorf et al. 2008; Richards 2008; Verhoeven et al. 2010).

So far, our understanding of ecological epigenetic effects on plants mostly originates from sexually reproducing species (especially a few model organisms such as Arabidopsis thaliana) through carefully controlled experiments (Bossdorf et al. 2010; Zhang et al. 2013; Schmid et al. 2018). However, in many ecosystems, plants with clonality is widespread and successfully dominant (Verhoeven and Preite 2014; Douhovnikoff and Dodd 2015; González et al. 2016). Clonal plants that largely rely on asexual reproduction often lack variation-generating mechanisms of meiotic recombination and segregation, leading to genetic monomorphism and reduced potential for genetically based adaptation (Verhoeven and Preite 2014). Therefore, epigenetic contribution is assumed to be particularly important in ecological processes of such clonal plants, as clonal propagation can circumvent some epigenetic resetting during meiosis, facilitating faithful persistence and transmission of DNA methylation states (Verhoeven and Preite 2014; González et al. 2016). Nevertheless, natural epigenetic variation in clonal plant populations and its relationships with genetic and phenotypic variation have remained little studied (Johnson and Tricker 2010; Grativol et al. 2012; Richards et al. 2012; Latzel et al. 2013).

To explore the role of epigenetic variation in natural populations of clonal plants, we investigated phenotypic, genetic and epigenetic variation of ten populations of an introduced clonal herb Hydrocotyle vulgaris in China by using amplified fragment length polymorphism (AFLP) and methylation-sensitive amplified polymorphism (MSAP) markers. Specifically, we addressed the following questions. (1) How much genetic and epigenetic variation exist in $H$. vulgaris populations? (2) Are intra- and interpopulation epigenetic variations independent from genetic variations? (3) To what extent do intra- and inter-population epigenetic and genetic variations contribute to phenotypic variation?

\section{Materials and methods}

\section{The species}

Hydrocotyle vulgaris L. (Araliaceae) is a perennial clonal herb and commonly distributed from semi-moist to wet conditions (Dong et al. 2015; Haslam 1988). It can form plagiotropic stems along which each node has the capacity of producing a ramet consisting of a petiolate leaf and adventitious roots (Dong et al. 2013). The species relies on vegetative propagation via stem fragments to form large clones and spread widely (Dong et al. 2015; Liu et al. 2014). Hydrocotyle vulgaris is native in Europe and was introduced to China in the 1990 s as an ornamental plant (Liu et al. 2014). Due to high phenotypic plasticity, rapid clonal reproduction, strong adaptability, and exclusion of other native species, it is considered potentially invasive in China (Dong et al. 2015; Liu et al. 2014; Miao et al. 2011).

\section{Sampling}

From June to August, 2016, we collected 128 samples of $H$. vulgaris from ten sites, with one population for each site (Table 1). In each population, sampling points were at least $5 \mathrm{~m}$ apart. To ensure that all samples were at the same developmental stage, we selected the mature ramet nearest to the sampling point and its adjacent four interconnected ramets as a sample (Appendix 1).

\section{Molecular analysis}

\section{DNA extraction}

For each sample, the leaf of the mature ramet nearest to the sampling point was collected and dried by silica gel. Total 
Table 1 Location, sample size and genotypes for the ten populations of Hydrocotyle vulgaris

\begin{tabular}{llllll}
\hline Pop. ID & Location & Coordinates & $\mathrm{N}$ & $\mathrm{G}$ & Genotype ID \\
\hline SZ & Shenzhen & $22^{\circ} 33^{\prime} 25^{\prime \prime} \mathrm{N}, 114^{\circ} 3^{\prime} 3^{\prime \prime} \mathrm{E}$ & 10 & 1 & $\mathrm{G} 3(10)$ \\
WZ & Wenzhou & $27^{\circ} 58^{\prime} 29^{\prime \prime} \mathrm{N}, 120^{\circ} 45^{\prime} 38^{\prime \prime} \mathrm{E}$ & 15 & 5 & $\mathrm{G} 3(8), \mathrm{G} 17(1), \mathrm{G} 18(3), \mathrm{G} 19(2), \mathrm{G} 20(1)$ \\
LS & Lishui & $28^{\circ} 23^{\prime} 11^{\prime \prime} \mathrm{N}, 19^{\circ} 49^{\prime} 9^{\prime \prime} \mathrm{E}$ & 10 & 2 & $\mathrm{G} 3(9), \mathrm{G} 5(1)$ \\
$\mathrm{TZ}$ & Taizhou & $28^{\circ} 40^{\prime} 22^{\prime \prime} \mathrm{N}, 121^{\circ} 25^{\prime} 37^{\prime \prime} \mathrm{E}$ & 15 & 4 & $\mathrm{G} 3(12), \mathrm{G} 13(1), \mathrm{G} 14(1), \mathrm{G} 15(1)$ \\
$\mathrm{CQ}$ & Chongqing & $29^{\circ} 33^{\prime} 34^{\prime \prime} \mathrm{N}, 106^{\circ} 37^{\prime} 41^{\prime \prime} \mathrm{E}$ & 9 & 4 & $\mathrm{G} 3(6), \mathrm{G} 1(1), \mathrm{G} 2(1), \mathrm{G} 9(1)$ \\
$\mathrm{NB}$ & Ningbo & $29^{\circ} 53^{\prime} 50^{\prime \prime} \mathrm{N}, 121^{\circ} 33^{\prime} 32^{\prime \prime} \mathrm{E}$ & 15 & 4 & $\mathrm{G} 3(12), \mathrm{G} 6(1), \mathrm{G} 11(1), \mathrm{G} 12(1)$ \\
$\mathrm{HZ}$ & Hangzhou & $30^{\circ} 18^{\prime} 48^{\prime \prime} \mathrm{N}, 120^{\circ} 23^{\prime} 25^{\prime \prime} \mathrm{E}$ & 13 & 4 & $\mathrm{G} 3(10), \mathrm{G} 4(1), \mathrm{G} 5(1), \mathrm{G} 8(1)$ \\
WH & Wuhan & $30^{\circ} 32^{\prime} 55^{\prime \prime} \mathrm{N}, 114^{\circ} 25^{\prime} 10^{\prime \prime} \mathrm{E}$ & 10 & 3 & $\mathrm{G} 3(7), \mathrm{G} 7(2), \mathrm{G} 16(1)$ \\
$\mathrm{JX}$ & Jiaxing & $30^{\circ} 41^{\prime} 55^{\prime \prime} \mathrm{N}, 120^{\circ} 46^{\prime} 28^{\prime \prime} \mathrm{E}$ & 21 & 1 & $\mathrm{G} 3(21)$ \\
$\mathrm{SH}$ & Shanghai & $31^{\circ} 8^{\prime} 58^{\prime \prime} \mathrm{N}, 121^{\circ} 26^{\prime} 30^{\prime \prime} \mathrm{E}$ & 10 & 1 & $\mathrm{G} 3(10)$ \\
\hline
\end{tabular}

Genotype ID G1-G20 represent the codes of the 20 different genotypes, numbers in the parentheses means number of ramets sharing the genotype

$N$ number of ramets sampled in each population, $G$ number of genotypes genomic DNA from $30 \mathrm{mg}$ of the dry leaf was extracted using Dingguo Plant Genomic DNA Kit (Beijing, China). Then the concentration and purity of the extracted DNAs were detected via a NanoDrop 2000c UV-Vis Spectrophotometer (Thermo scientific, MA, USA), and visualised by $1 \%$ agarose gel electrophoresis. After that, the DNAs were diluted to $20 \mathrm{ng} / \mu \mathrm{L}$ as the starting material for AFLP and MSAP analyses.

\section{AFLP analysis}

AFLP (amplified fragment length polymorphism) genotyping was carried out according to the original protocol of Vos et al. (1995) with several modifications (Appendix 2). Six selective amplification primer pairs (E-AGG/M-CAA, EACA/M-CAA, E-AAC/M-CAG, E-ACT/M-CAC, E-AAC/ M-CTA, and E-AGA/M-CCA) yielded clear polymorphism and generated about 50 well-separated peaks (for each primer pair) in 50-500 bp were screened from 120 primer pairs based on a subset of eight samples (Zhang et al. 2010). The fragment size of selective PCR products was assessed by ABI 3100 automated Genetic Analyser (Applied Biosystems, CA, USA). To estimate the error rate in AFLP genotyping, we randomly chose 24 DNA samples to duplicate the AFLP procedure.

\section{MSAP analysis}

MSAP was used to detect differences in DNA methylation status of samples. MSAP is a modification of the AFLP, which replaces $M s e$ I of AFLP by a pair of isoschizomeric restriction enzymes, HpaII and $M s p \mathrm{I}$, as frequent cutters (Herrera and Bazaga 2010). Both of these two enzymes recognise the sequence of CCGG but show different sensitivities to methylation states of external or internal cytosine residues (Cervera et al. 2002; McClelland et al. 1994;
Reyna-Lopez et al. 1997). Similarly, we chose six selective amplification primer pairs (E-AGT/H-TAT, E-AGT/HTTC, E-ATC/H-TGA, E-AAC/H-TCG, E-ATG/H-TGA, and E-ACA/H-TGA) from 140 primer combinations, using the same DNA samples for screening AFLP primer pairs. To estimate the error rate in MSAP genotyping, we randomly chose 24 DNA samples to duplicate the MSAP procedure.

\section{Scoring}

The raw molecular data (including replicates) were imported into Genemarker software version 2.2.0 (SoftGenetics LLC ${ }^{\circledR}$, State College, PA, USA) according to different primer combinations. We determined bin positions (i.e. locus) within the fragment-length (size) range of 54-500 bp for AFLP and 61-500 bp for MSAP analysis, respectively. Binary matrices that ' 1 ' denoted present peaks and ' 0 ' denoted absence were generated by the software automatically. After exporting them in Excel 2010 (Microsoft, WA, USA), we modified each binary matrix by checking it against the AFLP or MSAP profile by eyes.

For AFLP, the error rate was estimated for each primer combination by comparing the binary matrix of the 24 samples and their replicates, calculated as $100 \times$ (number of discordant scores on two independent analyses)/(number of scored markers $\times$ number of individuals) (Bonin et al. 2004; Herrera and Bazaga 2011). For MSAP, the error rate of HpaII $\left(e_{\mathrm{Hpa}}\right)$ and $\mathrm{MspI}\left(e_{\mathrm{Msp}}\right)$ scores were calculated separately for each primer combination based on the same method. Overall, the error rates for AFLP, MSAP with HpaII and MSAP with MspI were $0.34 \%, 0.77 \%$ and $0.53 \%$, respectively (Appendices 3 and 4).

In the binary matrix of MSAP patterns, presence of both EcoRI/HpaII and EcoRI/MspI digestions (pattern 1/1) denoted an unmethylated state of corresponding locus $\left(5^{\prime}\right.$ - 
CCGG target), presence of only one of EcoRI/HpaII (1/0) or EcoRI/MspI (0/1) products was considered as external cytosine hemimethylated or internal cytosine fully methylated, and absence of both EcoRI/HpaII and EcoRI/MspI (0/ $0)$ products represented an uninformative state (Gao et al. 2010; Pérez Figueroa 2013). Using msap package (Pérez Figueroa 2013), each locus was classified as either a methylation-susceptible locus (MSL) or a non-methylated locus (NML) by estimating whether the observed proportion of discordant HpaII-MspI scores across all samples exceeded an error rate-based threshold (i.e. ERT; if <ERT, defined as NML; if >ERT, defined as MSL) (Herrera and Bazaga 2010). The threshold was specific for each primer combination, calculated as $e_{\mathrm{Hpa}}+e_{\mathrm{Msp}}-2 e_{\mathrm{Hpa}} e_{\mathrm{Msp}}$, and ranged from 0.0075 to 0.0227 in this study (Appendix 4). Due to the fact that the banding pattern of NML depends exclusively on sequence changes at the restriction target, NML are used to assess genetic variation and MSL are used to assess epigenetic variation (Cervera et al. 2002; Pérez Figueroa 2013). Therefore, we transformed MSL data into a binary matrix where ' 0 ' represented HPA + /MSP + and HPA-/MSP-, and ' 1 ' represented HPA + /MSP- and HPA-/ $\mathrm{MSP}+$ for further analyses.

\section{Phenotypic trait measurement}

The two ramets adjacent to the ramet whose leaf was used for molecular analysis (Appendix 1) were collected to measure leaf area (LI-3100, Li-Cor, Lincoln, NE, USA), petiole length, internode length, and leaf chlorophyll content (SPAD-502, Konika Minolta, Osaka, Japan). Moreover, after drying at $70{ }^{\circ} \mathrm{C}$ for $48 \mathrm{~h}$, we weighed biomass of leaf and internode, respectively. Specific leaf area was calculated as leaf area divided by corresponding leaf dry mass, and specific internode length as internode length divided by corresponding internode dry mass. Mean petiole length, internode length, leaf area, specific leaf area, specific internode length and chlorophyll content were used as phenotypic traits.

\section{Statistical analysis}

Firstly, we determined clonal membership of each sample with GenoType (Meirmans and Tienderen 2004). After that, genetic and epigenetic diversity parameters of each population were quantified using Popgen32 software as (i) percentage of polymorphic loci $\left(\mathrm{PLP}_{\text {gen }}\right)$ and epiloci $\left(\mathrm{PLP}_{\mathrm{epi}}\right)$, (ii) Nei's gene diversity ( $h_{\text {gen }}$ and $h_{\text {epi }}$ ) and (iii) Shannon's information index of diversity $\left(I_{\mathrm{gen}}\right.$ and $I_{\mathrm{epi}}$ ). The variation of phenotypic traits (mean petiole length, internode length, leaf area, specific leaf area, specific internode length and leaf chlorophyll content) of each population was estimated as coefficient of variation $(\mathrm{CV}=100 \times$ standard deviation/ mean).
We used regression analyses to test the relationships between intra-population epigenetic and genetic diversity and between intra-population genetic diversity and phenotypic variation using SPSS 19.0 (SPSS, Chicago, IL, USA). We conducted Mantel tests to explore the correlations between inter-population epigenetic distance and genetic distance and between inter-population genetic distance and phenotypic differentiation with GenAlEx 6.5 (Peakall and Smouse 2012). For these analyses, data of all 128 samples were used. To examine the relationships between intra-population epigenetic diversity and phenotypic variation by regression analysis and between inter-population epigenetic distance and phenotypic differentiation by Mantel test, we used a subset of the data with only 105 samples having the same genotype (dominant genotype; see Table 1) based on AFLP markers to eliminate potential effects of genetic variation.

For Mantel tests, we calculated Euclidean distance of each of the six phenotypic traits (mean petiole length, internode length, leaf area, specific leaf area, specific internode length and leaf chlorophyll content) between populations as measures of inter-population phenotypic differentiation with NTSYS-pc (version 2.1, Exeter software, Setauket, USA). The significance of the correlations for Mantel tests was determined through the one-tail test after 999 permutations in GenAlEx 6.5.

\section{Results}

\section{Genetic and epigenetic diversity}

Based on data of all 128 samples, AFLP analysis revealed 317 markers, of which $25(7.89 \%)$ were polymorphic. The error rate for the six primer combinations ranged from 0.15 to $0.57 \%$, with the total error rate of $0.34 \%$ (Appendix 3 ). We distinguished 20 genotypes from all of the 128 individuals, among which a single widespread clone accounted for $82.03 \%(n=105)$ of the total samples and dominated in all ten populations, whereas 16 genotypes were each represented by only one single sample (Table 1). For genetic parameters of different populations, the percentage of polymorphic loci $\left(\mathrm{PLP}_{\text {gen }}\right)$ ranged from 0 (SZ, JX and $\mathrm{SH}$ ) to $2.21 \%$ (LS). Nei's gene diversity $\left(h_{\text {gen }}\right)$ ranged from 0 (SZ, JX and SH) to 0.0022 (LS), and Shannon's diversity index $\left(I_{\text {gen }}\right)$ ranged from 0 (SZ, JX and SH) to 0.0045 (LS) (Table 2).

Based on data of all 128 samples, the six primer combinations assayed in the MSAP analysis produced 468 clear and reproducible fragments ('loci' hereafter), of which 50 (10.68\%) were methylation-susceptible and 418 (89.32\%) were non-methylated. However, polymorphism level of methylation-susceptible loci (MSL, 46\%) was substantially higher than that of non-methylated loci (NML, 21\%) (Appendix 4). Among populations, the percentage of 
Table 2 Intra-population (A) genetic, (B) epigenetic and (C) phenotypic variation of Hydrocotyle vulgaris

\begin{tabular}{|c|c|c|c|c|c|c|}
\hline \multirow[b]{2}{*}{ Pop. ID } & \multicolumn{3}{|c|}{ (A) Genetic variation } & \multicolumn{3}{|c|}{ (B) Epigenetic variation } \\
\hline & $\operatorname{PLP}_{\text {gen }}(\%)$ & $h_{\text {gen }}$ & $I_{\mathrm{gen}}$ & $\operatorname{PLP}_{\text {epi }}(\%)$ & $h_{\text {epi }}$ & $I_{\text {epi }}$ \\
\hline $\mathrm{SZ}$ & 0 & 0 & 0 & 4.00 & 0.0074 & 0.0130 \\
\hline WZ & 1.26 & 0.0016 & 0.0031 & 8.00 & 0.0290 & 0.0433 \\
\hline LS & 2.21 & 0.0022 & 0.0045 & 22.00 & 0.0964 & 0.1386 \\
\hline $\mathrm{TZ}$ & 1.26 & 0.0012 & 0.0025 & 6.00 & 0.0186 & 0.0292 \\
\hline CQ & 1.58 & 0.0017 & 0.0035 & 8.00 & 0.0275 & 0.0412 \\
\hline NB & 1.58 & 0.0010 & 0.0023 & 8.00 & 0.0259 & 0.0389 \\
\hline $\mathrm{HZ}$ & 1.58 & 0.0016 & 0.0033 & 16.00 & 0.0425 & 0.0666 \\
\hline WH & 0.95 & 0.0018 & 0.0031 & 8.00 & 0.0255 & 0.0383 \\
\hline JX & 0 & 0 & 0 & 16.00 & 0.0556 & 0.0832 \\
\hline SH & 0 & 0 & 0 & 8.00 & 0.0096 & 0.0189 \\
\hline
\end{tabular}

(C) Phenotypic variation

\begin{tabular}{llllllc} 
Pop. ID CV of petiole & $\begin{array}{l}\text { CV of internode } \\
\text { length }(\%)\end{array}$ & $\begin{array}{l}\text { CV of leaf } \\
\text { area }(\%)\end{array}$ & $\begin{array}{l}\text { CV of } \\
\text { SIL }(\%)\end{array}$ & $\begin{array}{l}\text { CV of } \\
\text { SLA }(\%)\end{array}$ & $\begin{array}{l}\text { CV of } \\
\text { chlorophyll }(\%)\end{array}$ \\
\hline SZ & 21.69 & 40.94 & 45.18 & 40.58 & 17.40 & 7.93 \\
WZ & 23.65 & 25.38 & 34.78 & 38.22 & 17.75 & 9.21 \\
LS & 30.42 & 41.23 & 53.10 & 47.74 & 24.51 & 10.30 \\
TZ & 27.45 & 21.03 & 34.05 & 52.07 & 26.63 & 19.48 \\
CQ & 28.82 & 44.67 & 17.39 & 58.18 & 24.36 & 12.28 \\
NB & 27.61 & 32.75 & 48.37 & 47.41 & 15.51 & 6.88 \\
HZ & 32.47 & 30.67 & 44.04 & 46.52 & 17.82 & 5.89 \\
WH & 33.27 & 15.94 & 27.92 & 54.28 & 12.77 & 6.93 \\
JX & 53.50 & 33.20 & 68.16 & 38.97 & 39.67 & 12.59 \\
SH & 37.82 & 48.41 & 38.40 & 76.66 & 21.26 & 12.85 \\
\hline
\end{tabular}

Population ID is described as in Table 1

PLP $(\%)$ percentage of polymorphic loci $\left(\mathrm{PLP}_{\mathrm{gen}}\right)$ and epiloci $\left(\mathrm{PLP}_{\mathrm{epi}}\right), h$ Nei's genetic diversity $\left(h_{\mathrm{gen}}\right)$ and epigenetic diversity $\left(h_{\mathrm{epi}}\right), I$ Shannon's information index of genetic diversity $\left(I_{\mathrm{gen}}\right)$ and epigenetic diversity $\left(I_{\text {epi }}\right)$, SIL specific internode length, SLA specific leaf area polymorphic MSL (PLP ${ }_{\text {epi }}$ ) ranged from $4.00 \%$ (SZ) to $22.00 \%$ (LS). Nei's diversity index for epigenetic variation $\left(h_{\text {epi }}\right)$ ranged from 0.007 (SZ) to $0.096(\mathrm{LS})$, and Shannon's diversity index for epigenetic variation $\left(I_{\mathrm{epi}}\right)$ ranged from 0.013 (SZ) to 0.139 (LS) (Table 2). Our results suggest that the level of epigenetic diversity is higher than that of genetic diversity in $H$. vulgaris populations.

\section{Correlations between intra-population genetic, epigenetic and phenotypic variation}

We found a concave-up relationship between intrapopulation genetic diversity and epigenetic diversity (for PLP $_{\text {epi }}: R^{2}=0.57, P=0.054$; for $h_{\text {epi }}: R^{2}=0.57, P=0.053$; for $I_{\mathrm{epi}}: R^{2}=0.66, P=0.023$, Fig. 1 ), suggesting that epigenetic diversity reached a nadir at the intermediate value of genetic diversity, and increased at higher and lower values. This pattern still held when the population with excess epigenetic variation but low genetic variation (the data point on the top left in Fig. 1) was excluded (Appendix 5).
There was no correlation between intra-population genetic diversity and phenotypic variation (Appendix 6). When genetic variation was controlled (i.e. using samples of the dominant genotype), intra-population epigenetic diversity was positively correlated with CV of petiole length (for $\mathrm{PLP}_{\mathrm{epi}}: R^{2}$ $=0.71, P=0.002$; for $h_{\text {epi }}: R^{2}=0.61, P=0.008$; for $I_{\text {epi }}: R^{2}$ $=0.66, P=0.005$, Fig. 2a-c) and showed a quadratic (concave) relationship with both CV of leaf area (for PLP $\operatorname{Pp}_{\text {epi }}: R^{2}=$ $0.62, P=0.035$; for $h_{\text {epi }}: R^{2}=0.49, P=0.098$; for $I_{\text {epi }}: R^{2}=$ $0.54, P=0.066$, Fig. 2d-f) and CV of specific leaf area (for $\mathrm{PLP}_{\mathrm{epi}}: R^{2}=0.61, P=0.036$; for $h_{\mathrm{epi}}: R^{2}=0.78, P=0.005$; for $I_{\text {epi }}: R^{2}=0.74, P=0.009$, Fig. $2 \mathrm{~g}-\mathrm{i}$ ).

\section{Correlations between inter-population genetic, epigenetic and phenotypic distance}

Mantel test revealed a marginally positive correlation between inter-population genetic and epigenetic distance ( $r=0.475, P=0.068$; Fig. 3a). Inter-population genetic distance had no correlation with phenotypic differentiation 


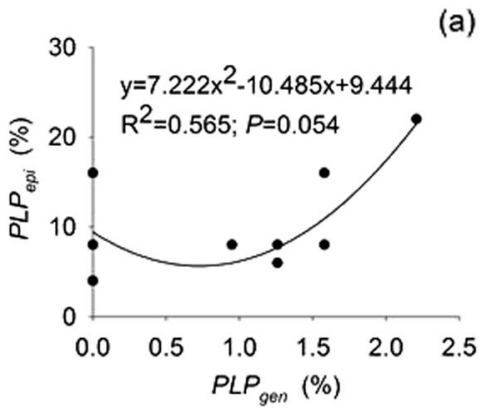

(a)

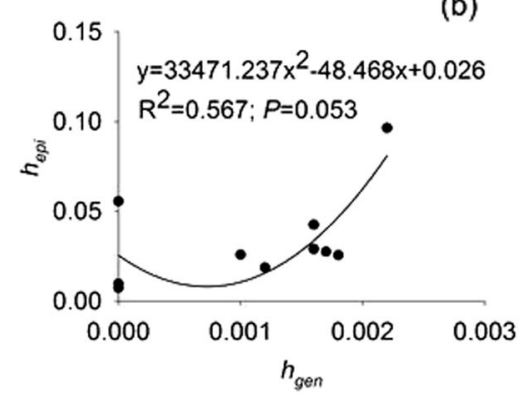

(b)

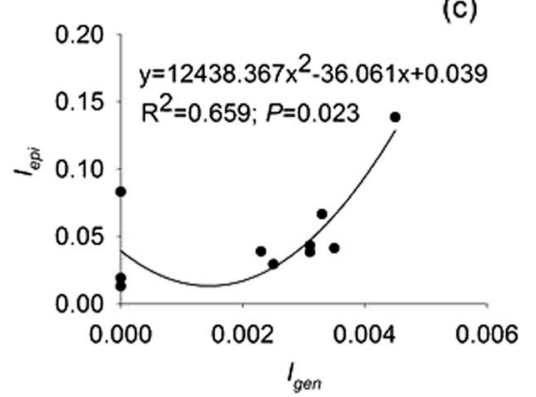

Fig. 1 Relationships between intra-population genetic diversity and epigenetic diversity using data of all 128 samples $(n=10)$
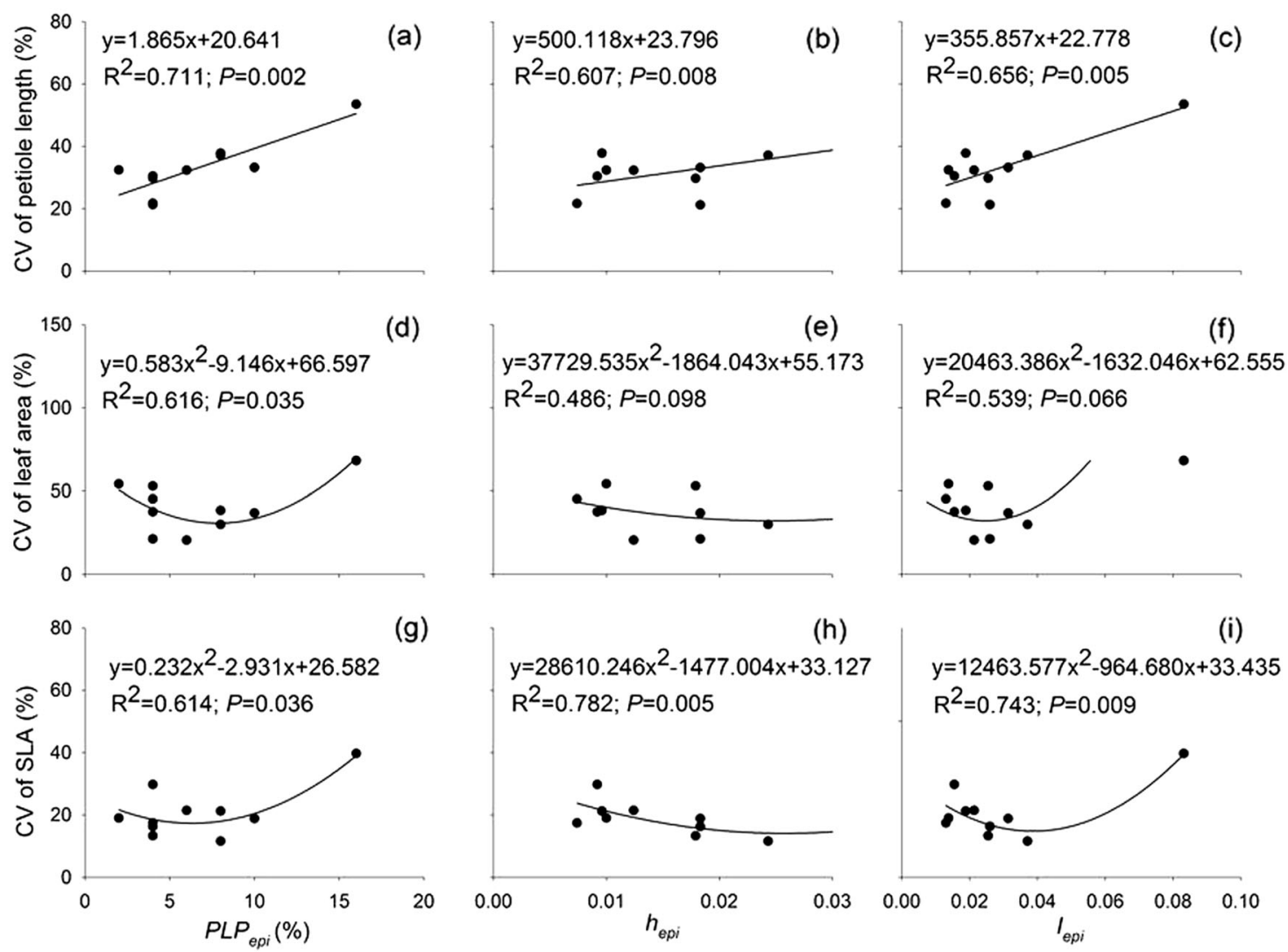

Fig. 2 Relationships between intra-population epigenetic diversity and phenotypic variation of $H$. vulgaris populations using a subset of the data with 105 samples having the same genotype $(n=10)$

(Appendix 7). However, when effects of genetic variation were controlled by using samples of the dominant genotype, inter-population epigenetic distance was positively correlated with leaf area differentiation $(r=0.405, P=0.043$; Fig. 3b).

\section{Discussion}

\section{Genetic and epigenetic diversity}

Our study revealed that genetic diversity of the $H$. vulgaris populations in China was very low, and a widespread clone accounting for $82 \%$ of the total individuals dominated in all ten populations. This result is similar to several highly invasive clonal plants exhibiting genetic uniformity in their introduced ranges, such as water hyacinth (Eichhornia crassipes), alligator weed (Alternanthera philoxeroides) and Japanese knotweed (Fallopia japonica) (Hollingsworth and Bailey 2000; Geng et al. 2007; Zhang et al. 2010; Richards et al. 2012; Zhang et al. 2016). The lack of genetic variation may be attributed to several reasons such as limited sexual reproduction, high degree of clonal propagation, successive nested bottlenecks during invasive expansion, and unfavourable environmental conditions for seed germination (Vaughn et al. 2007; Zhang et al. 2010). 
Fig. 3 Mantel test for the correlation a between interpopulation epigenetic and genetic differentiation using data of all 128 samples, and $\mathbf{b}$ between inter-population epigenetic distance and phenotypic differentiation using a subset of the data with only 105 samples having the same genotype
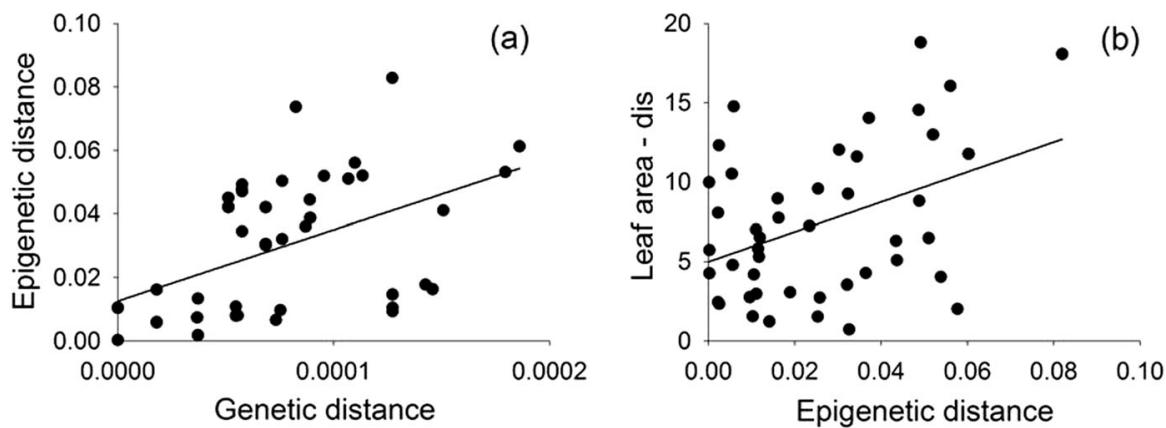

Our study showed that epigenetic diversity of the $H$. vulgaris populations was significantly higher than genetic diversity, similar to several previous studies (Gao et al. 2010; Herrera and Bazaga 2010; Lira-Medeiros et al. 2010; Richards et al. 2012). Epigenetic changes are now widely known to be triggered by different environmental conditions (Cervera et al. 2002; Verhoeven et al. 2010; Richards 2011; Preite et al. 2015). Moreover, there is also another possible origin for epigenetic changes-spontaneous epimutation, which occurs at higher frequencies than mutations of DNA sequence when populations undergo a genetic bottleneck (Richards 2008; Richards et al. 2010; Zhang et al. 2010).

\section{Correlations between epigenetic and genetic variation}

The relationship between genetic and epigenetic variation in wild populations is still questionable, attracting the attention of many researchers (Massicotte et al. 2011; Schulz et al. 2014; Foust et al. 2016). Some studies have revealed that epigenetic variation is completely uncoupled from genetic variation, indicating that the population-specific selection could act on both genetic and epigenetic variation independently (Riddle and Richards 2002; Verhoeven et al. 2010; Avramidou et al. 2015). Such independency may be ascribed to random epigenetic drift-dramatic spontaneous alterations of epigenetic marks occurring during mitotic propagation or epimutations (Richards 2008; Banta and Richards 2018).

In contrast, we found concave-up relationships between intra-population genetic diversity and epigenetic diversity of $H$. vulgaris, and a marginally positive correlation between inter-population genetic and epigenetic distance. Similarly, a significant correlation between genetic and epigenetic variation has been detected in e.g. Hordeum brevisubulatum, Viola elatior, Arabidopsis thaliana and Spartina alterniflora, suggesting that epigenetic variation is at least in part a downstream, subsidiary effect of genetic variation (Shan et al. 2012; Schulz et al. 2014; Dubin et al. 2015; Kawakatsu et al. 2016; Robertson et al. 2017).
Moreover, methylation-based epigenetic distance of Viola cazorlensis populations was found to be associated with adaptive genetic divergence, indicating that epigenetic differentiation could be driven by variable selections (Herrera and Bazaga 2010). Such epigenetic modifications might be caused by several cis and trans genetic information that contributes to the acquisition and retention of epigenetic marks at specific loci (Riddle and Richards 2002; Richards 2008; Herrera and Bazaga 2011; Richards et al. 2017).

\section{Correlations of phenotypic variation with genetic and epigenetic variation}

Classically, DNA sequence variation is considered to determine phenotypic variation (Grativol et al. 2012; Bian et al. 2013; Hirsch et al. 2013). An association between genetic (AFLP) markers and phenotypic traits was detected in some of previous studies (e.g. Herrera and Bazaga 2008; Medrano et al. 2014). However, contrary to our expectation, there is no correlation of intra- or inter- population genetic variation with phenotypic variation of $H$. vulgaris. It may be attributed to the low proportion of the AFLP markers that are positioned within gene sequences regulating the expression of some specific traits (Caballero et al. 2013).

More recently, it was found that DNA methylation can regulate gene expression levels through interfering with transcription and influencing the formation of transcriptionally silent heterochromatin, further leading to the changes in phenotypes (Zilberman et al. 2007; Jablonka and Raz 2009; Herrera and Bazaga 2010; Grativol et al. 2012). In our study, when we used data of all 128 samples to investigate the relationship between epigenetic variation and phenotypic variation, PLP $_{\text {epi }}$ was significantly positively related to $\mathrm{CV}$ of petiole length and marginally significantly $(P<0.1)$ related to $\mathrm{CV}$ of leaf area (Appendix 8 ), while neither $h_{\text {epi }}$ nor $I_{\text {epi }}$ was significantly associated with intra-population variation of any of the six phenotypic traits measured (data not shown). Moreover, there was no correlation between inter-population epigenetic distance and phenotypic differentiation (data not shown). After taking advantage of clonal organisms with a low level of genetic 
variation by using a subset of the data with 105 samples having the same genotype (i.e. the dominant genotype) based on AFLP markers to tease out genetic effects, we found that intra-population epigenetic diversity was significantly associated with phenotypic variation in petiole length, leaf area and specific leaf area, and inter-population epigenetic distance was correlated with leaf area differentiation. These results suggest that epigenetic variation may independently play a role in shaping plant phenotypes, and genetic effects may act in an additive way with opposite direction when accounting for epigenetic influences on phenotypic variation.

According to Medrano et al. (2014), individual traits tend to be associated exclusively with either AFLP or MSAP markers involved in genomic regions, and the probability of such relatedness is roughly triple times higher in MSAP than in AFLP markers. This may be the reason that individual differences in the methylation status are more easily detected to have a relationship with phenotypic changes within natural plant populations. There is also a similar finding in Solanum ruiz-lealii, showing that different flower phenotypes between individuals were generated through the changes of cytosine methylation polymorphism rather than the genetic variation pattern (Marfil et al. 2009). The DNA methylation differentiation among populations likely results from different environmental factors in different sites as specific epiloci may be an important component of response to different habitats in plants, and previous studies have indicated phenotypic traits could change through epigenetic alterations in response to changing environments (Richards et al. 2012; Medrano et al. 2014; Foust et al. 2016). Such a mechanism has been proposed to extend the plasticity of a single genotype to take advantage of a wider ecological niche (Spens and Douhovnikoff 2016). Moreover, epimutations could trigger phenotypic variation at equilibrium due to different epigenetic states, with higher frequency of occurrence than genetic mutations, serving as a faster source to enhance the adaptive possibilities of asexual or lowdiversity species (Richards et al. 2012; Banta and Richards 2018).

\section{Conclusions}

Clonal plants with a low level of genetic diversity (e.g. $H$. vulgaris in our study) offer advantages in studying causes and consequences of epigenetic variation, since confounding effects of genetic (DNA sequence) variation on plants can be controlled by clonal identity (Verhoeven and Preite 2014; Douhovnikoff and Dodd 2015). In this study, H. vulgaris populations exhibited extremely low genetic diversity but high epigenetic diversity. Even though epigenetic variation was partly under genetic control, it might independently shape phenotypic variation both within and among populations. Such epigenetically related phenotypic variation may compensate for shortfalls in genetic diversity and facilitate the wide distribution of clonality in natural environments, possibly acting as an additional system to accelerate evolution of clonal plant populations (Kalisz and Purugganan 2004; Schrey et al. 2012; Douhovnikoff and Dodd 2015). However, AFLP and MSAP methods only offer a limited number of anonymous loci, unable to reveal causality between genetic/epigenetic and phenotypic variation (Paun et al. 2018). To further acquire insight into functional characterisation from genetic/epigenetic loci to associated gene expression effects to phenotypes, greater resolution methods such as QTL mapping and reduced representation bisulphite sequencing could be used (Verhoeven and Preite 2014; Foust et al. 2016; Richards et al. 2017).

\section{Data archiving}

Data available from the Dryad Digital Repository: https:// doi.org/10.5061/dryad.vk5c064.

Acknowledgements We thank Xiang-Qi Bu and Shao-Ke Li for their assistance, and two anonymous reviewers for their insightful comments. The work was supported by NSFC $(31570413,31870610)$.

\section{Compliance with ethical standards}

Conflict of interest The authors declare no conflict of interests.

Publisher's note: Springer Nature remains neutral with regard to jurisdictional claims in published maps and institutional affiliations.

\section{References}

Avramidou EV, Ganopoulos IV, Doulis AG, Tsaftaris AS, Aravanopoulos FA (2015) Beyond population genetics: natural epigenetic variation in wild cherry (Prunus avium). Tree Genet Genomes 95:1-9

Banta JA, Richards CL (2018) Quantitative epigenetics and evolution. Heredity 121:210-224

Bian RJ, Nie DD, Fu X, Zhou XL, Gao Y, Bai ZJ et al. (2013) Adaptational significance of variations in DNA methylation in clonal plant Hierochloe glabra (Poaceae) in heterogeneous habitats. Aust J Bot 61:274-282

Bonin A, Bellemain E, Eidesen PB, Pompanon F, Brochmann C, Taberlet $P$ (2004) How to track and assess genotyping errors in population genetics studies. Mol Ecol 13:3261-3273

Bossdorf O, Arcuri D, Richards CL, Pigliucci M (2010) Experimental alteration of DNA methylation affects the phenotypic plasticity of ecologically relevant traits in Arabidopsis thaliana. Evol Ecol 24:541-553

Bossdorf O, Richards CL, Pigliucci M (2008) Epigenetics for ecologists. Ecol Lett 11:106-115

Boyko A, Kovalchuk I (2011) Genome instability and epigenetic modification-heritable responses to environmental stress? Curr Opin Plant Biol 14:260-266

Busconi M, Colli L, Sánchez RA, Santaella M, Pascual MD, Santana $O$ et al. (2015) AFLP and MS-AFLP analysis of the variation 
within Saffron Crocus (Crocus sativus L.) germplasm. PLoS One 4:e123434

Caballero A, Garcia-Pereira MJ, Quesada H (2013) Genomic distribution of AFLP markers relative to gene locations for different eukaryotic species. BMC Genom 14:528

Cervera MT, Ruiz-Garcia L, Martinez-Zapater JM (2002) Analysis of DNA methylation in Arabidopsis thaliana based on methylationsensitive AFLP markers. Mol Genet Genom 268:543-552

Dong BC, Wang JZ, Liu RH, Zhang MX, Luo FL, Yu FH (2015) Soil heterogeneity affects ramet placement of Hydrocotyle vulgaris. $\mathrm{J}$ Plant Ecol 8:91-100

Dong BC, Wang JZ, Liu RH, Zhang MX, Yu FH (2013) Effects of heterogeneous competitor distribution and ramet aggregation on the growth and size structure of a clonal plant. PLOS ONE 8: e68557

Douhovnikoff V, Dodd RS (2015) Epigenetics: a potential mechanism for clonal plant success. Plant Ecol 216:227-233

Dubin MJ, Zhang P, Meng D, Remigereau M, Osborne EJ, Casale FP et al. (2015) DNA methylation variation in Arabidopsis has a genetic basis and shows evidence of local adaptation. eLife 4:1-23

Foust CM, Preite V, Schrey AW, Alvarez M, Robertson MH, Verhoeven KJF et al. (2016) Genetic and epigenetic differences associated with environmental gradients in replicate populations of two salt marsh perennials. Mol Ecol 25:1639-1652

Gao LX, Geng YP, Li B, Chen JK, Yang J (2010) Genome-wide DNA methylation alterations of Alternanthera philoxeroides in natural and manipulated habitats: implications for epigenetic regulation of rapid responses to environmental fluctuation and phenotypic variation. Plant Cell Environ 33:1820-1827

Geng YP, Pan XY, Xu CY, Zhang WJ, Li B, Chen JK et al. (2007) Phenotypic plasticity rather than locally adapted ecotypes allows the invasive alligator weed to colonize a wide range of habitats. Biol Invasions 9:245-256

González APR, Chrtek J, Dobrev PI, Dumalasová V, Fehrer J, Mráz P et al. (2016) Stress-induced memory alters growth of clonal offspring of white clover (Trifolium repens). Am J Bot 103:1567-1574

Grativol C, Hemerly AS, Ferreira PCG (2012) Genetic and epigenetic regulation of stress responses in natural plant populations. Biochim Biophys Acta 1819:176-185

Haslam SM (1988) River plants of Western Europe: the macrophytic vegetation of watercourses of the European Economic Community. Cambridge University Press, New York, NY

Herman JJ, Spencer HG, Donohue K, Sultan SE (2014) How stable 'should' epigenetic modifications be? Insights from adaptive plasticity and bet hedging. Evolution 68:632-643

Herman JJ, Sultan SE (2016) DNA methylation mediates genetic variation for adaptive transgenerational plasticity. Proc R Soc B: Biol Sci 283:20160988

Herrera CM, Bazaga P (2008) Population-genomic approach reveals adaptive floral divergence in discrete populations of a hawk moth —pollinated violet. Mol Ecol 17:5378-5390

Herrera CM, Bazaga P (2010) Epigenetic differentiation and relationship to adaptive genetic divergence in discrete populations of the violet Viola cazorlensis. New Phytol 187:867-876

Herrera CM, Bazaga P (2011) Untangling individual variation in natural populations: ecological, genetic and epigenetic correlates of long-term inequality in herbivory. Mol Ecol 20:1675-1688

Hirsch S, Baumberger R, Grossniklaus U (2013) Epigenetic variation, inheritance, and selection in plant populations. Cold Spring Harb Symp Quant Biol 77:97-104

Hollingsworth ML, Bailey JP (2000) Evidence for massive clonal growth in the invasive weed Fallopia japonica (Japanese Knotweed). Bot J Linn Soc 133:463-472
Jablonka E, Raz G (2009) Transgenerational epigenetic inheritance: prevalence, mechanisms, and implications for the study of heredity and evolution. Q Rev Biol 84:131-176

Johnson LJ, Tricker PJ (2010) Epigenomic plasticity within populations: its evolutionary significance and potential. Heredity 105:113-121

Kalisz S, Purugganan MD (2004) Epialleles via DNA methylation: consequences for plant evolution. Trends Ecol Evol 19:309-314

Kawakatsu T, Huang SC, Jupe F, Sasaki E, Schmitz RJ, Urich MA et al. (2016) Epigenomic diversity in a global collection of Arabidopsis thaliana Accessions. Cell 166:492-505

Latzel V, Allan E, Silveira AB, Colot V, Fischer M, Bossdorf O (2013) Epigenetic diversity increases the productivity and stability of plant populations. Nat Commun 4:2875

Lira-Medeiros CF, Parisod C, Fernandes RA, Mata CS, Cardoso MA, Ferreira PCG (2010) Epigenetic variation in mangrove plants occurring in contrasting natural environment. PLoS ONE 5: e10326

Liu RH, Chen QW, Dong BC, Yu FH (2014) Effects of vegetative propagule pressure on the establishment of an introduced clonal plant, Hydrocotyle vulgaris. Sci Rep 4:5507

Marfil CF, Camadro EL, Masuelli RW (2009) Phenotypic instability and epigenetic variability in a diploid potato of hybrid origin, Solanum ruiz-lealii. BMC Plant Biol 9:21

Massicotte R, Whitelaw E, Angers B (2011) DNA methylation: a source of random variation in natural populations. Epigenetics 6:421-427

McClelland M, Nelson M, Raschke E (1994) Effect of site-specific modification on restriction endonucleases and DNA modification methyltransferases. Nucleic Acids Res 22:3640-3659

Medrano M, Herrera CM, Bazaga P (2014) Epigenetic variation predicts regional and local intraspecific functional diversity in a perennial herb. Mol Ecol 23:4926-4938

Meirmans PG, Tienderen PHV (2004) Genotype and genodive: two programs for the analysis of genetic diversity of asexual organisms. Mol Ecol Notes 4:792-794

Miao LH, Ji MC, Wang YY, Qiao DD, Chen YC (2011) Study on invasion risk of Hydrocotyle vulgaris as an alien species in wetlands. J Zhejiang Univ 37:425-431

Paun O, Verhoeven KJF, Richards CL (2018) Opportunities and limitations of reduced representation bisulfite sequencing in plant ecological epigenomics. New Phytol 221:738-742

Peakall R, Smouse PE (2012) GenAlEx 6.5: genetic analysis in Excel. Population genetic software for teaching and research-an update. Bioinformatics 28:2537-2539

Preite V, Snoek LB, Oplaat C, Biere A, van der Putten WH, Verhoeven KJF (2015) The epigenetic footprint of poleward rangeexpanding plants in apomictic dandelions. Mol Ecol 24:4406-4418

Pérez Figueroa A (2013) MSAP: a tool for the statistical analysis of methylation-sensitive amplified polymorphism data. Mol Ecol Resour 13:522-527

Rapp RA, Wendel JF (2005) Epigenetics and plant evolution. New Phytol 168:81-91

Reyna-Lopez GE, Simpson J, Ruiz-Herrera J (1997) Differences in DNA methylation patterns are detectable during the dimorphic transition of fungi by amplification of restriction polymorphisms. Mol Gen Genet MGG 253:703-710

Richards CL, Alonso C, Becker C, Bossdorf O, Bucher E, ColoméTatché $\mathrm{M}$ et al. (2017) Ecological plant epigenetics: evidence from model and non-model species, and the way forward. Ecol Lett 20:1576-1590

Richards CL, Bossdorf O, Verhoeven KJF (2010) Understanding natural epigenetic variation. New Phytol 187:562-564 
Richards CL, Schrey AW, Pigliucci M (2012) Invasion of diverse habitats by few Japanese knotweed genotypes is correlated with epigenetic differentiation. Ecol Lett 15:1016-1025

Richards EJ (2006) Inherited epigenetic variation-revisiting soft inheritance. Nat Rev Genet 7:395-401

Richards EJ (2008) Population epigenetics. Curr Opin Genet Dev $18: 221-226$

Richards EJ (2011) Natural epigenetic variation in plant species: a view from the field. Curr Opin Plant Biol 14:204-209

Riddle NC, Richards EJ (2002) The control of natural variation in cytosine methylation in Arabidopsis. Genetics 162:355-363

Robertson MH, Schrey AW, Shayter A, Moss CJ, Richards CL (2017) Genetic and epigenetic variation in Spartina alterniflora following the Deepwater Horizon oil spill. Evol Appl 10:792-801

Schmid MW, Heichinger C, Coman Schmid D, Guthörl D, Gagliardini V, Bruggmann R et al. (2018) Contribution of epigenetic variation to adaptation in Arabidopsis. Nat Commun 9:4446

Schrey AW, Coon CAC, Grispo MT, Awad M, Imboma T, McCoy ED et al. (2012) Epigenetic variation may compensate for decreased genetic variation with introductions: a case study using house sparrows (Passer domesticus) on two continents. Genet Res Int 2012:1-7

Schulz B, Eckstein RL, Durka W (2014) Epigenetic variation reflects dynamic habitat conditions in a rare floodplain herb. Mol Ecol 23:3523-3537

Shan XH, Li YD, Liu XM, Wu Y, Zhang MZ, Guo WL et al. (2012) Comparative analyses of genetic/epigenetic diversities and structures in a wild barley species (Hordeum brevisubulatum) using MSAP, SSAP and AFLP. Genet Mol Res 11:2749-2759

Spens AE, Douhovnikoff V (2016) Epigenetic variation within Phragmites australis among lineages, genotypes, and ramets. Biol Invasions 18:2457-2462
Thiebaut F, Hemerly AS, Ferreira PCG (2019) A role for epigenetic regulation in the adaptation and stress responses of non-model plants. Front Plant Sci 10:246

Vaughn MW, Bulski A, Colot V, Doerge RW, Martienssen RA, Tanurdzi MôLZ et al. (2007) Epigenetic natural variation in Arabidopsis thaliana. PLoS Biol 5:e174

Verhoeven KJF, Jansen JJ, van Dijk PJ, Biere A (2010) Stressinduced DNA methylation changes and their heritability in asexual dandelions. New Phytol 185:1108-1118

Verhoeven KJF, Preite V (2014) Epigenetic variation in asexually reproducing organisms. Evolution 68:644-655

Vos P, Hogers R, Bleeker M, Reijans M, Van de Lee T, Hornes M et al. (1995) AFLP: a new technique for DNA fingerprinting. Nucleic Acids Res 23:4407-4414

Zhang YY, Fischer M, Colot V, Bossdorf O (2013) Epigenetic variation creates potential for evolution of plant phenotypic plasticity. New Phytol 197:314-322

Zhang YY, Parepa M, Fischer M, Bossdorf O (2016) Epigenetics of colonizing species? A study of Japanese Knotweed in central Europe. Invasion genetics: the baker and stebbins legacy, WileyBlackwell, Oxford

Zhang YY, Zhang DY, Barrett SCH (2010) Genetic uniformity characterizes the invasive spread of water hyacinth (Eichhornia crassipes), a clonal aquatic plant. Mol Ecol 19:1774-1786

Zilberman D, Gehring M, Tran RK, Ballinger T, Henikoff S (2007) Genome-wide analysis of Arabidopsis thaliana DNA methylation uncovers an interdependence between methylation and transcription. Nat Genet 39:61-69 